\title{
BISTABLE BEHAVIOUR OF THE NEW SHALLOW THERMAL DONOR IN ALUMINUM DOPED SILICON
}

\author{
P. KACZOR, M. GODLEWSKI \\ Institute of Physics, Polish Academy of Sciences \\ Al. Lotników 32/46, 02-668 Warszawa, Poland \\ AND T. Gregorkiewicz \\ Van der Waals-Zeeman Laboratorium, University of Amsterdam \\ Vackelnierstraat 65, 1018 XE Amsterdam, The Netherlands
}

\begin{abstract}
In the present study a new bistable shallow thermal donor in aluminum doped silicon was investigated by means of the Fourier transform infrared spectroscopy. The temperature dependence of the photo-conversion into the metastable state was established and some hints for the origin of the metastability were given.
\end{abstract}

PACS numbers: 71.55 . Ht, 78.50. Ge

\section{Introduction}

Thermal donors (TDs) in Czochralski-grown silicon belong to the most studied defect systems in solids. They appear after prolonged thermal annealing of the oxygen-rich silicon in the temperature range of about $350-470^{\circ} \mathrm{C}$. It is quite well established that the TDs are formed in a process of oxygen clustering, but the formation mechanism itself (simple diffusion cannot account for it), as well as, the influence of co-doping remain still unclear. Also despite the extensive research done over last three decades no definitive models exist which could explain their microscopic structure [1-2].

The infrared absorption studies contributed significantly to our present knowledge on properties of TDs [2]. It has been established that two distinct spectral series can be related to TDs, proving them to be double donors (well described by the effective mass theory), with ground state energies of approximately $60 \mathrm{meV}$ $\left(\mathrm{TD}^{0} / \mathrm{TD}^{+}\right)$and $150 \mathrm{meV}\left(\mathrm{TD}^{+} / \mathrm{TD}^{++}\right)$below the conduction band. Relatively recent investigations of photothermal ionization spectroscopy [3] revealed a series of lines being a signature of a new single donor species called shallow thermal donors (STDs). They are generated simultaneously to TD and they have ground state energies of about $30 \mathrm{meV}$. 
In the earlier work the creation of STDs was attributed either to the material properties (highly resistive $\mathrm{Si}$ ) [3] or to specific impurities like nitrogen [4] or $\mathrm{Al}$ in high concentrations [5]. Recently, comparative studies done with FTIR and ENDOR techniques have helped to find out that the existence of STDs in the heat treated silicon is a more general phenomenon - it does not depend on the particular dopant. It was concluded that only the rate of STD-creation depends on the doping level [6-8]. It was also shown that in Al doped material a new shallow thermal donor appears with the activation energy of about $39.5 \mathrm{meV}$. The properties of the new STD center are discussed in the present work. We show that it has a bistable character depending on cooling and illumination procedures.

\section{Experimental results and discussion}

The sample (labelled W38) used in the experiment was cut off of a high quality commercial Czochralski-grown low-carbon silicon material (WASO grade) doped with aluminum $\left(4 \times 10^{15} \mathrm{~cm}^{-3}\right)$. After the standard initial heat treatment used for oxygen dispersion, the sample was subsequently annealed at $470^{\circ} \mathrm{C}$ for 11.5 hours in order to get measurable STD concentration. Then the infrared

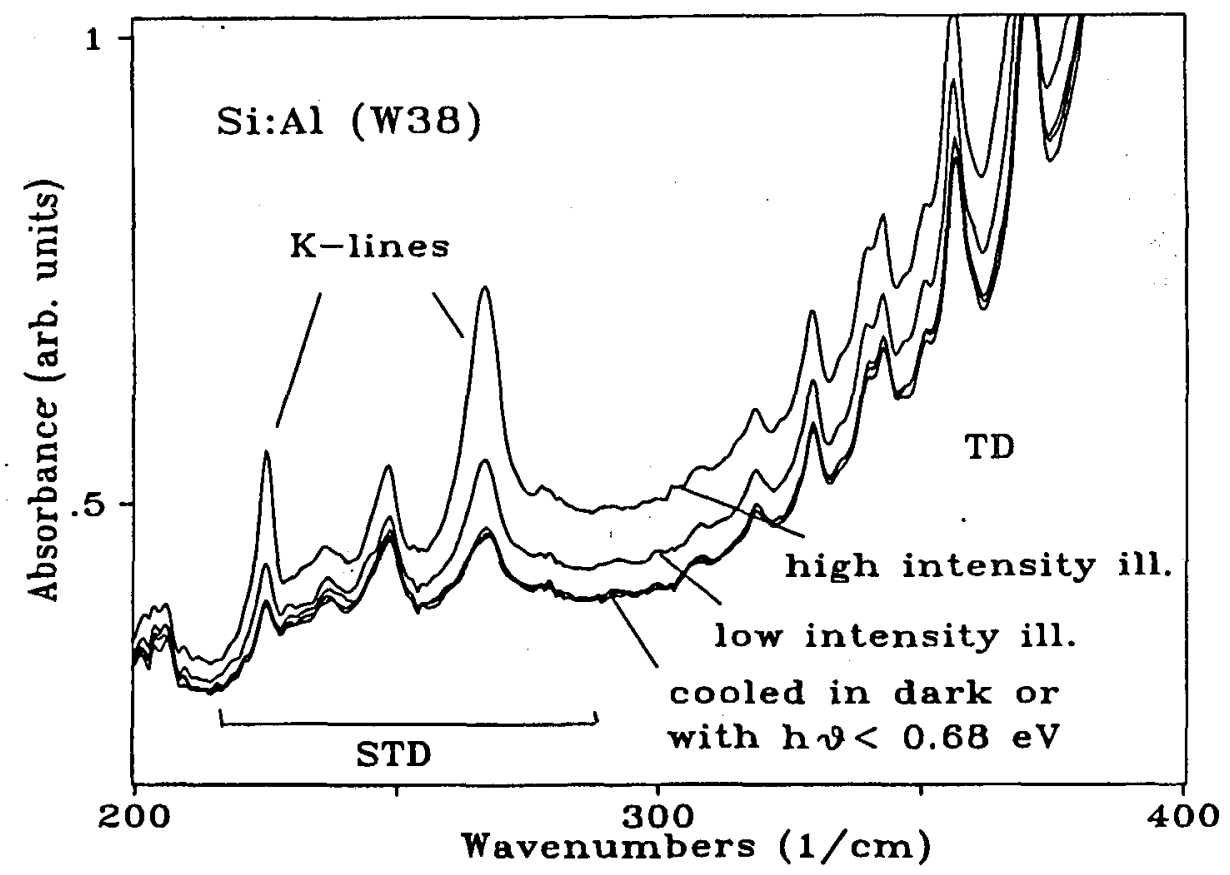

Fig. 1. STD spectra in Al doped silicon. The spectrum of the sample illuminated at $T=130 \mathrm{~K}$ for $1 \mathrm{~h}$ is compared to the spectrum of the sample cooled in the dark or illuminated with various filters. Also the effect of the reduced intensity of the illuminating light is shown. 


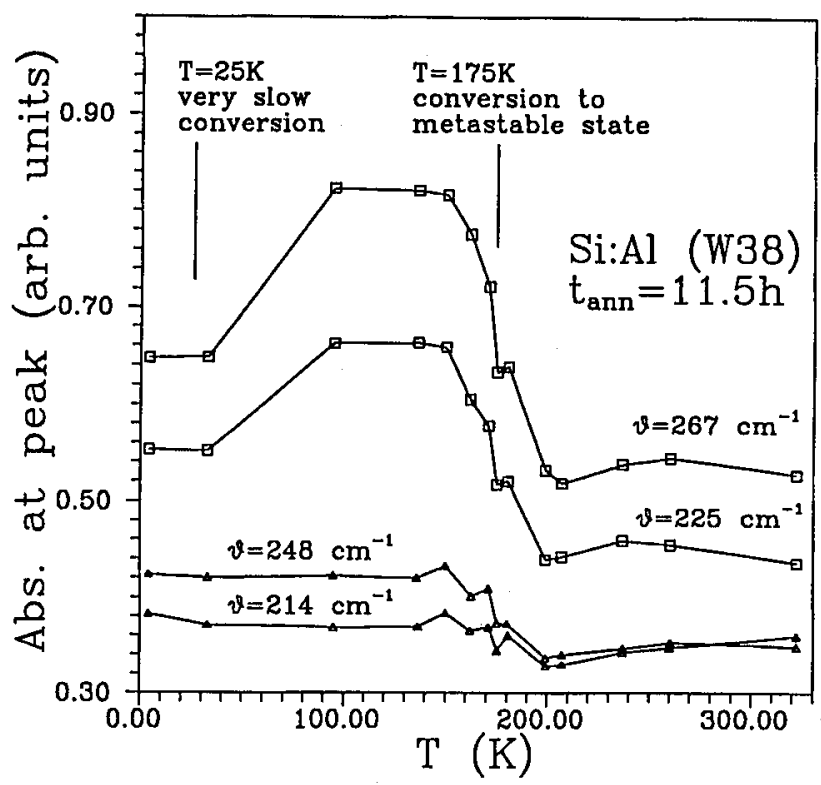

Fig. 2. Temperature dependence of the photo-conversion into the metastable new shallow thermal donor (squares). The photo-conversion starts at about $190 \mathrm{~K}$ and is most effective at liquid nitrogen temperatures. Illumination at low temperatures does not canse the photo-conversion. For comparison, the temperature dependence of the base line (at $\nu=214 \mathrm{~cm}^{-1}$ ) and of the intensity of the most pronounced STD-line (at $\nu=248 \mathrm{~cm}^{-1}$ ) is shown (triangels).

absorption measurements were performed at liquid helium temperature with the Bomem FTIR spectrometer.

Two absorption lines much stronger than other STD-lines have been found when the sample was illuminated with near band-gap light during cooling and labelled $K$-lines (at $225 \mathrm{~cm}^{-1}$ for $1 s-2 p_{0}$ transition and at $267 \mathrm{~cm}^{-1}$ for $1 \mathrm{~s}-2 p_{+}$ transition). The intensity of these lines was considerably reduced if the sample was cooled in the darkness [7] (Fig. 1).

The lines also appear if the sample is cooled in the dark, illuminated at selected temperatures and subsequently cooled in the dark to $4.2 \mathrm{~K}$. Due to the weak intensity of the illuminating light a long illumination time was necessary ( 1 hour). It has been found that such exposure to the illumination at temperatures of about 80-190 K gives rise to the metastable population of the new STD (Fig. 2). The lower the temperature the greater the strength of $K$-lines is - suggesting the existence of a barrier preventing a recapture of the STD-electrons on a deeper level.

On the other hand at low temperatures $(10-30 \mathrm{~K})$ the photo-conversion into the metastable state does not practically occur even if very strong illumination is used. The photo-conversion has been found to be less efficient when the light of 
lower intensity was applied under the same illumination conditions (Fig. 1).

The described metastable behaviour is very similar to that observed for NL10 spectrum in Si:Al [9] and resembles the shallow-deep bistability behaviour characteristic for localised centres with large lattice relaxation like e.g. the DX-centres in AlGaAs.

At the present stage of the experimental work it will be too early to speculate about the nature of the observed bistability of the new STD in Si:Al. It is known that the first deepest TDs exhibit bistable behaviour of the DX-type [10] and it is also established that the interstitial $\mathrm{Al}$ in $\mathrm{Si}$ (which may be generated during the TD-formation [11]) is a bistable centre [12]. Further investigations are needed to reveal the mechanism of the bistability and the microscopic structure of the defect.

\section{References}

[1] L.C. Kimmerling, in: Proc. of the Symposium on Oxygen, Carbon, Hydrogen and Nitrogen in Crystalline Silicon, Materials Research Society Symposia Proceedings, Eds. J.C. Mikkelsen Jr., S.J. Pearton, J.W. Corbett, S.J. Pennycook, Vol. 59, MRS, Pittsburg 1986, p. 83.

[2] P. Wagner, J. Hage, Appl. Phys. Lett. A 49, 123 (1989).

[3] J.A. Griffin, H. Navarro, J. Weber, L. Genzel, J.T. Borenstein, J.W. Corbett, L.C. Snyder, J. Phys. C 19, L579 (1986).

[4] M. Suezawa, K. Sumino, H. Harada, T. Abe, Jpn. J. Appl. Phys. 25, L859 (1986).

[5] M. Claybourn, R.C. Newman, Mater. Sci. Forum 38-41, 613 (1989).

[6] K. Kopalko, P. Kaczor, M. Godlewski, T. Grégorkiewicz, Acta Phys. Pol. A 80, 345 (1991).

[7] P. Kaczor, K. Kopalko, M. Godlewski, T. Gregorkiewicz, Acta Phys. Pol. A 82, 677 (1992).

[8] T. Gregorkiewicz, H.H.P.Th. Bekman, C.A.J. Ammerlaan, Phys. Rev. B 41, 628 (1990).

[9] H.H.P.Th. Bekman, T. Gregorkiewicz, C.A.J. Ammerlaan, Phys. Rev. Lett. 61, 227 (1988).

[10] L.M. Makarenko, V.P. Markevich, L.I. Murin, Sov. Phys. Semicond. 19, 1192 (1985).

[11] R.C. Newman, J. Phys C 18, L967 (1985).

[13] J.R. Troxell, A.P. Chatterje, G.D. Watkins, L.C. Kimmerling, Phys. Rev. B 19, 5336 (1979). 